\title{
Sucht und Kriminalität
}

\author{
Norbert Leygraf
}

Online publiziert: 29. Januar 2015

(C) Springer-Verlag Berlin Heidelberg 2015

Süchtiges und delinquentes Verhalten scheinen eng miteinander verwandt $\mathrm{zu}$ sein, jedenfalls treten sie häufig gemeinsam auf. Dies ist zunächst nur ein korrelativer und kein ursächlicher Zusammenhang, zumindest nicht in dem Sinne, dass das eine Verhalten das andere bedingen würde, auch wenn in Strafverfahren oft versucht wird, eine derartige Kausalbeziehung herzustellen. Geht es hierbei um die kriminogene Wirkung der akuten Berauschung, war meist Alkohol im Spiel, dessen enthemmende und Aggressionshandlungen fördernde Wirkung nicht nur alltäglich bekannt, sondern wissenschaftlich belegt ist. Dies gilt für andere Suchtmittel nicht in gleicher Weise, insbesondere nicht für Cannabis, was auch ein Grund dafür sein dürfte, dass im Strafvollzug auf einen Alkoholkonsum zumeist sehr viel rigider reagiert wird als auf einen Cannabiskonsum. Die weite Verbreitung von Cannabis und anderen Drogen im Strafvollzug dürfte im Übrigen nicht alleine durch die spezifischen Probleme der Strafgefangenen bedingt sein, sondern weist auf die Bedeutung sozialer Rahmen- und Umgebungsbedingungen für einen solchen Konsum hin.

Neben der akuten Intoxikation sind v. a. die psychosozialen Folgeerscheinungen der Sucht von kriminogener Bedeutung. Dies betrifft Veränderungen der Persönlichkeit und der kognitiven Fähigkeiten, aber auch den suchtbedingten Verlust von Arbeit und prosozialen Kontakten und das Hineingeraten in ein subkulturelles Milieu. Hinzu kommen bei illegalen Drogen die im Vergleich zu Alkohol höheren Kosten, die zu Beschaffungsdelikten Anlass geben können.

Prof. Dr. N. Leygraf $(\bowtie)$

Institut für Forensische Psychiatrie, LVR-Klinikum Essen,

Universität Duisburg-Essen,

Essen, Deutschland

E-Mail: Norbert.Leygraf@uni-due.de
Aufgrund der vielfältigen Überschneidungen von psychiatrisch-psychologischen, sozialwissenschaftlichen und strafrechtlichen Aspekten sind die Zusammenhänge von Drogenkonsum und Kriminalität ein bevorzugtes Thema kriminologischer Forschung. Der Beitrag von Kreuzer gibt eine zusammenfassende Übersicht über die Ergebnisse bisheriger empirischer Untersuchungen, darunter auch eigener, über mehrere Jahrzehnte erfolgter Studien. Er beschreibt die Komplexität möglicher Einflussgrößen auf den biografischen Verlauf von Drogenkonsum und delinquentem Verhalten. So kann eine zunächst bestehende Drogenabhängigkeit in die (Beschaffungs-)Kriminalität führen; eine früh einsetzende kriminelle Karriere kann aber auch erst im weiteren Verlauf von einer zusätzlichen Drogenabhängigkeit begleitet sein. Zwischen diesen beiden Verlaufstypen gibt es einen weiten Bereich von Menschen, bei denen Suchtmittelkonsum und Delinquenz Ausdruck einer generellen Bereitschaft zu sozial abweichendem Verhalten darstellen. Der enge Zusammenhang zwischen illegalem Drogenkonsum und Straffälligkeit basiert v. a. darauf, dass ein solcher Konsum gehäuft in kriminalitätsbelasteten Milieus stattfindet; zumindest führt die Beschaffung der Drogen an ein solches Milieu heran. Zudem beginnen später Drogenabhängige ihren Konsum meist schon in einem frühen, besonders kriminalitätsgefährdeten Lebensalter.

Die Delinquenz von Drogenabhängigen lässt sich also nicht alleine auf ihre Suchtproblematik reduzieren. Somit erscheint es sinnvoll, auch im therapeutischen Umgang mit ihnen nicht nur auf suchtspezifische Aspekte zu fokussieren, sondern kriminal- bzw. sozialtherapeutische Maßnahmen einzubeziehen. Solche Programme, die u. a. auf die Stärkung sozialer und emotionaler Kompetenzen abzielen, sind im Strafvollzug weit etabliert, wenn auch wenig hinsichtlich ihrer Wirksamkeit erforscht. Der Beitrag von Gadek et al. informiert über die Ergebnisse einer Studie, in der die Effek- 
tivität eines solchen „Guppentrainings sozialer Kompetenzen“ bei einer Gruppe drogenabhängiger Strafgefangener überprüft wurde. Dabei zeigte sich zwar testpsychologisch eine Verbesserung der Selbstsicherheit und Selbstzufriedenheit, aber keine Veränderung in den problematischen Verhaltensmustern. Die Autoren diskutieren mögliche Hintergründe dieses etwas ernüchternden Ergebnisses und berichten über die Entwicklung eines modifizierten Behandlungskonzeptes, das sich derzeit in der Pilotphase befindet.

Ebenfalls ernüchternd sind schon seit vielen Jahren die Verlaufsdaten der Behandlung suchtkranker Straftäter in einer Maßregel gemäß $\S 64$ StGB. Gut die Hälfte dieser Unterbringungen wird als nicht weiter erfolgversprechend abgebrochen, zumeist ist zu diesem Zeitpunkt bereits mehr als ein Jahr (auch kostenintensiver) Maßregelbehandlung verstrichen. Hintergrund dieser Abbrüche sind oft Suchtmittelrückfälle, die in der Phase beginnender Vollzugslockerungen erfolgen. Dabei wird in der Maßregel weiterhin die therapeutische Idealvorstellung einer lebenslangen Abstinenz angestrebt, die in der allgemeinen Suchtbehandlung zugunsten realistischer, hierarchisch gestaffelter Zielvorstellungen abgelöst worden ist. Im Kontext solcher langfristiger Behandlungsplanungen haben sich auch medikamentöse Hilfemaßnahmen etabliert. Der Beitrag von Heilmann und Scherbaum gibt eine umfassende Übersicht über die derzeit gebräuchlichen medikamentösen Behandlungsstrategien und beschreibt Möglichkeiten, durch Substitution oder eine abstinenzunterstützende Medikation die bisherigen Behandlungsoptionen im Maßregelvollzug sinnvoll zu ergänzen und die Unterbringung effektiver zu gestalten. Gerade dieser besonders schwierig zu behandelnden Gruppe suchtkranker Straftäter sollten nicht aufgrund therapeutischer Idealvorstellungen realistische, in ihrer Wirksamkeit gut evaluierte Hilfen verweigert werden.
Die beiden folgenden Beiträge befassen sich mit der Behandlung und Rückfallprophylaxe bei einer jeweils recht speziellen Klientel. Krammer et al. untersuchten die Effekte eines schon seit Längerem im Behandlungsvollzug etablierten Gruppentrainings (,,Reasoning and Rehabilitation Revised“, R\&R2) bei einer Gruppe in der Schweiz inhaftierter Straftäterinnen. Nach Abschluss der 16 Gruppensitzungen zeigten sich positive Veränderungen $u$. a. im Hinblick auf ein stabileres Selbstwertgefühl und eine verbesserte Emotionsregulation; auch nahm eine von den Teilnehmerinnen wahrgenommene posttraumatische Belastungssymptomatik ab. Es bleibt jedoch abzuwarten, ob dies auch zu überdauernden Verhaltensänderungen und v. a. zu einer Senkung der Rückfalldelinquenz führt. Deutlich längerfristig angelegt ist die forensische Rehabilitation zuvor Sicherungsverwahrter, über die $V o \beta$ am Beispiel der Forensisch-Therapeutischen Ambulanz in Berlin berichtet. Die nach dem Urteil des Europäischen Gerichtshofs für Menschenrechte (EGMR) im Dezember 2009 verbreitete Sorge über die Entlassung als gefährlich eingeschätzter Sicherungsverwahrter hat sich als wenig begründet erwiesen. Der Beitrag von $V o \beta$ zeigt, dass sich auch diese Gruppe (ehemaliger) Straftäter bei Beachtung ihrer Besonderheiten erfolgreich in ein ambulantes, multiprofessionelles Nachsorgesetting integrieren lässt.

Nicht nur in Deutschland hat die Kriminalität jugendlicher Straftäter mit Migrationshintergrund einen hohen medialen Stellenwert; mögliche Hintergründe solcher Taten werden dabei meist wenig differenziert betrachtet. In den von Kilchmann et al. berichteten Ergebnissen einer vergleichenden Studie in der Schweiz wiesen delinquente Jugendliche mit Migrationshintergrund ein deutlich höheres Maß an familiären Belastungsfaktoren sowie eine niedrigere Quote erfolgreicher Schulverläufe auf, worauf bei Präventions- und Interventionsmaßnahmen verstärkt zu achten ist. 